\title{
Family data underused to track disease
}

For many people tracking their family history, the Web offers a wealth of information to aid their genealogical search. And, for the medically inclined, some online discussion forums focus specifically on tracking illnesses in families, such as Huntington's disease (HD).

"If you are tracking a family which carries $\mathrm{HD}$, please add your information here and consider joining our mailing list. My g-ggrandmother may be your g-g-aunt," Gina Heffernan writes on her site, HD Genealogy (http://hdgenealogy.org/).

Researchers also see value in family data. "I think we are seeing a pretty sharp return to family-based studies in human genetics," says David Goldstein, director of Duke University's Center for Human Genome Variation in Durham, North Carolina. For example, with the growing use of sequencing to explore disease risk, he says, "it becomes considerably more important to be able to evaluate the precise phenotypes of individuals that carry the variant of interest and also to assess its effects in relatives." Moreover, genetic history can reveal diseases based on multiple genes, whereas individual genotyping might reveal only traits based on single genes.

According to Joann Boughman, executive vice president of the American Society of Human Genetics, testing one person's genes gives some idea of potential health risks, but not as much as knowing that, say, a person's genetic history includes three people in the past two generations who died in their 50s of heart attacks. As Boughman says, "If I have that information, I have you pegged."

The company 23andMe in Mountain View,

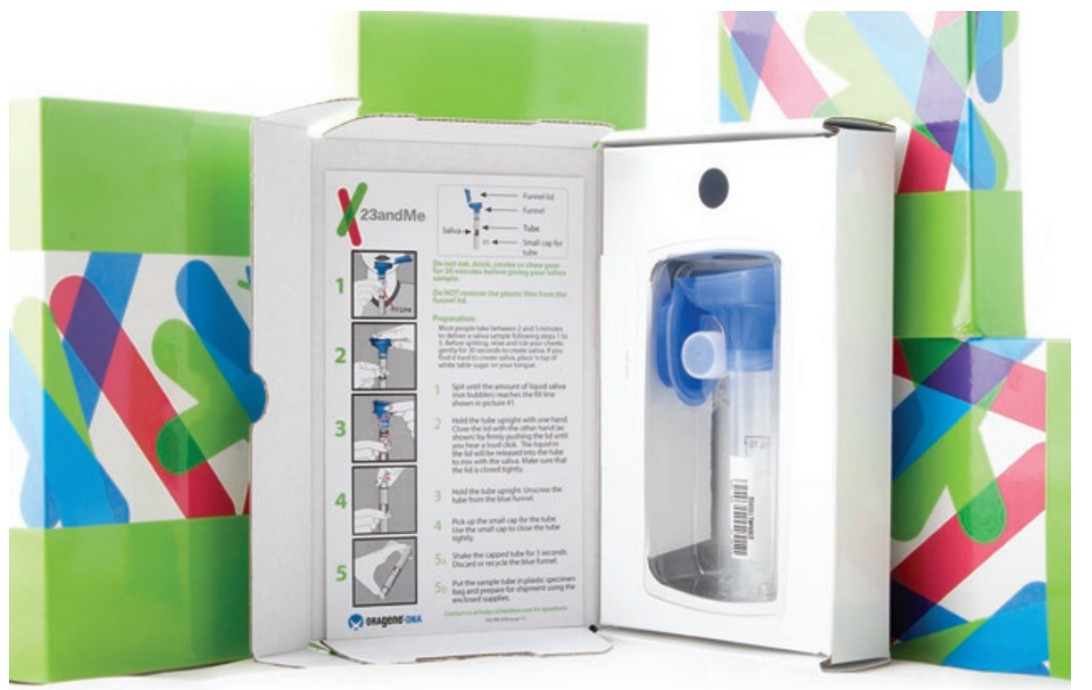

Spit kit: Home gene tests offer insights into disease.
California is trying to use genotypes across families to understand diseases, especially Parkinson's disease. In March 2009, this company joined forces with the Parkinson's Institute and Clinical Center in Sunnyvale, California and the New York-based Michael J. Fox Foundation with a goal of genotyping 10,000 people with the disease.

Researchers at 23andMe have been tracking Parkinson's in several families. One of the company's customers turned up homozygous for a mutation named G2019S in the LRRK2 gene. According to Lizzie Dorfman, former cohort manager for the 23andMe Parkinson's research program, "this mutation is fairly penetrant and uncommon. About half the people who even have one copy of it get Parkinson's, and people with two copies are very rare. It is an incredibly important mutation to study to understand Parkinson's disease." To further the research, 23 andMe offered free testing to this customer's family, and now the company has tested about 25 members across three generations.

Growing databases of genealogical information and increasing numbers of genotyped individuals could help track disease genes over generations. Nonetheless, much of the data goes uncollected, sometimes because of concerns over the need for added genetic counseling and potential regulatory issues related to medical testing.

Online sites that collect gigantic amounts of genealogical data include Ancestry.com and Family Tree DNA. For example, Ancestry. com includes 16 million family trees-but not necessarily health-related information. "Each user dictates what information they include in their tree, so really how useful a pedigree would be in regard to determining genetic diseases depends entirely on the member who created the tree," says Suzanne Campbell, a spokesperson for the site. "If they included a cause of death on an individual in their tree or attached an obituary or death certificate, that could be useful in understanding family propensity toward diseases." She adds, however, that this "is not the purpose" of the site.

Likewise, Family Tree DNA of Houston holds a database of almost 300,000 genotypes for genealogy and recently collaborated with Affymetrix of Santa Clara, California to use a DNA chip to match relatives across seven generations.

Although Family Tree DNA's latest genealogical product, the Family Finder test, includes more than half a million single nucleotide polymorphisms (SNPs), it intentionally omits any medically related ones. To include these medical SNPs, Bennett Greenspan, Family Tree DNA's president and chief executive officer points out that the statistics of any disease-related results would need to be carefully explained to a customer. "For that reason, we don't want to get involved in that controversy," he says.

In contrast, some sites aim at the very connection between genealogy and health. For instance, the American Society of Human Genetics and the Genetic Alliance created a website called Know Your Family Health History (www.talkhealthhistory.org/), which aims to provide "a comprehensive source of family health history information, along with helpful tools and resources, for patients, families and healthcare providers."

Using sequencing to help generate family histories, however, might face some challenges. In June, the US Food and Drug Administration sent out warning letters to five companies saying that the tests they offer require the agency's approval (See 'Genetic test kit oversight could prove a mixed blessing for research,' page 728).

For now, the Parkinson's disease project at 23andMe continues. "In the last year, we've identified hundreds of people who carry this rare mutation," Dorfman says. "Engaging with these people will enable many new avenues of research in Parkinson's disease, and this was achieved in large part by working with families."

Despite the ongoing research efforts, Boughman says bluntly, "family history is underutilized." 